\title{
IAU Office of Astronomy for Development: Task Force Children and School Education
}

\author{
Pedro Russo $^{1}$ and Edward Gomez ${ }^{2}$ \\ ${ }^{1}$ EU Universe Awareness - Leiden University, the Netherland \\ ${ }^{2}$ Las Cumbres Observatory Global Telescope Network \\ E-mail: russo@strw.leidenuniv.nl \& egomez@lcogt.net
}

\begin{abstract}
The main mission of the IAU OAD Task Force on Children and School Education is to support the implementation of the pre-tertiary education part of the IAU Strategic Plan 'Astronomy for Development'. In this presentation we will give an overview of the role and programme of the task force as well as a general discussion about the past, present and future IAU education activities and programmes.
\end{abstract}

\section{Introduction and Scope}

The task force on Astronomy for Children and Schools (TF2) for the IAU Office of Astronomy for Development drives activities related to using astronomy to inspire the very young and stimulate education, especially in Mathematics and Science. This task force looks at introducing astronomy in schools where there is little or no astronomy, and ensuring that the subject is used to positively influence the level of education development. Programmes for very young children, in the early childhood development stage, also fall within this task force. Examples of activities are educator training workshops; developing classroom resources; astronomy clubs in schools; etc.

\section{Work Plan}

TF2 is in charge of some organisational and community tasks in the framework of the IAU OAD activities. Organisational Tasks include: (i) supporting OAD Regional Nodes activities, (ii) facilitating interactions between pre-tertiary education stakeholders, (iii) working closely with the relevant IAU Commissions, and (iv) supporting the OAD fundraising. Community management level tasks include

- providing a small ( 2) number of core projects.

- providing input to the OAD database of astronomy education contacts (teachers, informal educators, etc)

- preparing, planning and supporting the implementation of an Annual Astronomy Education Strategy with on-going global/regional educational activities, opportunities for educational collaborations and other relevant information.

- issuing a call and managing a review committee for project proposals made to the OAD TF2 for endorsement and funding.

- delivering regular information (via OAD website, e-newsletter and social media, etc) about ongoing activities and opportunities, in astronomy education to volunteers, partner organisations and community 


\section{Core Projects}

Core Project 1 deals with a Peer Review Platform for astronomy educational resources. This platform will provide a method for people to submit their educational resources to review and obtain objective guidance on the resources, have successful resources published in a central repository and receive IAU approval/accreditation. Resources will be open and freely available to everyone and will be able to be submitted in any language. Resources will also be made available in many different formats - PDF (print quality and low-res), .odt, HTML, epub, mobi, etc. and will be syndicated through document sharing sites (OER, Issuu, Slideshare, other social media networks).

According to the 4R Resource Repository Model (Wiley 2000), resources need to follow the four criteria: Reuse (the right to reuse the content in its unaltered / verbatim form); Revise (the right to adapt, adjust, modify, or alter the content itself); Remix (the right to combine the original or revised content with other content to create something new; and Redistribute (the right to share copies of the original content, your revisions, or your remixes with others. The repository, currently under development, will need to follow one more criteria: Review. Peer review is essential for the sucess of the repository. The resources' content and quality will be reviewed by the community peers, with the aim of improving each resource and following a similiar approach as the peer-review process in the scientific community.

Core Project 2 is the AstroPack. The distribution of physical materials improves the engagement level with the community. Physical materials can inspire people in general, and young children in particular, in ways which online resources cannot. They also provide a physical reminder of the context they received them (e.g. at an astronomy workshop) and helps to sustain their engagement with astronomy. TF2 will manage a physical pack with physical astronomy resources, which can be delivered in a bundle for wide distribution by local representatives. The main idea is that the pack contents is provided by the community and will be curated by TF2 members. The distribution will be coordinated by the OAD and the first mail-drop is expected for early 2013.

\section{Projects Proposals}

A call for projects proposal was issued during 2012. These project proposals aligned with the IAU's 10 year strategic plan, with specific focus on Astronomy for Children and Schools. Some examples of proposals include teacher training and development; resource development and vetting; producing guidelines and support for using robotic telescopes for school projects; exploring distance learning options applicable to school level education; etc.

\section{References}

Wiley, D. 2000. The Instructional Use of Learning Objects. Bloomington, IN: AECT. 